Article

\title{
Approximation of CDF of Non-Central Chi-Square Distribution by Mean-Value Theorems for Integrals
}

\author{
Árpád Baricz ${ }^{1,2^{\mathbb{D}}}$, Dragana Jankov Maširević ${ }^{3}$ and Tibor K. Pogány and, $^{2,4}$ \\ 1 Department of Economics, Babeş-Bolyai University, 400591 Cluj-Napoca, Romania; \\ arpad.baricz@econ.ubbcluj.ro \\ 2 Institute of Applied Mathematics, Óbuda University, Bécsi út 96/b, 1034 Budapest, Hungary \\ 3 Department of Mathematics, University of Osijek, Trg Lj. Gaja 6, 31000 Osijek, Croatia; djankov@mathos.hr \\ 4 Faculty of Maritime Studies, University of Rijeka, Studentska 2, 51000 Rijeka, Croatia \\ * Correspondence: poganj@pfri.hr
}

Citation: Baricz, Á.; Jankov

Maširević, D.; Pogány, T.K.

Approximation of CDF of

Non-Central Chi-Square Distribution

by Mean-Value Theorems for

Integrals. Mathematics 2021, 9, 129.

https://doi.org/10.3390/math9020129

Received: 9 December 2020

Accepted: 4 January 2021

Published: 8 January 2021

Publisher's Note: MDPI stays neutral with regard to jurisdictional clai$\mathrm{ms}$ in published maps and institutional affiliations.

Copyright: $\odot 2021$ by the authors. Licensee MDPI, Basel, Switzerland. This article is an open access article distributed under the terms and conditions of the Creative Commons Attribution (CC BY) license (https:// creativecommons.org/licenses/by/ $4.0 /)$.

\begin{abstract}
The cumulative distribution function of the non-central chi-square distribution $\chi_{n}^{\prime 2}(\lambda)$ of $n$ degrees of freedom possesses an integral representation. Here we rewrite this integral in terms of a lower incomplete gamma function applying two of the second mean-value theorems for definite integrals, which are of Bonnet type and Okamura's variant of the du Bois-Reymond theorem. Related results are exposed concerning the small argument cases in cumulative distribution function (CDF) and their asymptotic behavior near the origin.
\end{abstract}

Keywords: non-central $\chi^{2}$ distribution; second mean-value theorem for definite integrals; modified Bessel function of the first kind; Marcum $Q$-function; lower incomplete gamma function

MSC: Primary: 26A24, 62E17; Secondary: 33C10, 60E99

\section{Introduction with Historical Notes and Motivation}

The non-central $\chi^{2}$ distribution with $n \in \mathbb{N}$ degrees of freedom (in general, $n$ can be a non-negative real number, see ([1] (p. 436), [2]) and non-centrality parameter $\lambda>0$ is usually denoted by $\chi_{n}^{\prime 2}(\lambda)$ (see, e.g., [1] (p. 433)) and it is one of the most applied distributions: it is important in calculating the power function of some statistical tests [3], precisely in approximating to the power of $\chi^{2}$-tests applied to contingency tables (goodness of fit tests) ([1] (p. 467)); it frequently occurs in finance, estimation and decision theory and time series analysis $[4,5]$ and can also be regarded as a generalized Rayleigh distribution ([1] (p. 435)) in which case it is used in mathematical physics; when it is used in communication theory then we call the appropriate complementary cumulative distribution function the generalized Marcum $Q$-function and the non-centrality parameter is interpreted as a signal-to-noise ratio [1].

The beginnings of the research that led up to the model and finally results in the $\chi_{n}^{2}$ distribution, which is the zero non-centrality parameter case of non-central $\chi_{n}^{\prime 2}(\lambda)$, that is $\chi_{n}^{2} \equiv \chi_{n}^{\prime 2}(0)$, can be located around the middle of the 19th century. More precisely there are two main opinions exposed: firstly, the influential work by Lancaster [6] who attributed certain preliminary results to Bienaymé in ([7] (p. 58)) (never mentioning normal distribution), which are in fact the same as what Karl Pearson did to earn his tables [8]. It is not surprising, Bienaymé's interest in the sum of random squares and the related distribution of errors; namely, we should have in mind his celebrated result on the linearity of variance of a sum of independent random variables called the Bienaymé formula. Lancaster proceeded then to Helmert, who in $[9,10]$ derived that which we understand in modern notation the $\chi_{n}^{2}$ probability density function (PDF). Finally, Lancaster joined Kruskal [11], suggesting to call the distribution by Helmert's name. 
However, Sheynin [12], Plackett [13] and especially Kendall [14] have mentioned the contribution of the applied mathematician and physicists Ernst Abbe, who has published in his venia docendi thesis [15] in Jena, 1863, the $\chi^{2}$ distribution's PDF ([12] (p. 1004)). It is also worth mentioning that Helmert himself never explicitly mentioned this distribution as Abbe's result in this manner, but several times quoted the "modified Abbe's criterion" in geodetic literature ([12] (p. 1004)). Kendall emphasizes Abbe's priority (agreeing with Sheynin) and wrote a laudatio to his work regarding the derivation of the PDF of $\chi^{2}$ distribution (in a contemporary notation) ([14] (p. 311, Equation (11))), preceding Helmert for at least twelve years.

A random variable (rv) $\xi$ possesses non-central $\chi^{2}$ distribution, which we signify with $\xi \sim \chi_{n}^{\prime 2}(\lambda)$ if the associated probability density function is ([4] (p. 396, Equation (1.7)))

$$
f_{n, \lambda}(x)=\frac{1}{2} \mathrm{e}^{-(x+\lambda) / 2}\left(\frac{x}{\lambda}\right)^{(n-2) / 4} I_{n / 2-1}(\sqrt{\lambda x}), \quad \lambda>0, x>0 ; n \in \mathbb{N},
$$

where $I_{v}$ stands for the modified Bessel function of the first kind of order $v([16]$ (p. 77)) and has the power series representation ([17] (p. 375, Equation (9.6.10)))

$$
I_{v}(z)=\sum_{k=0}^{\infty} \frac{1}{\Gamma(v+k+1) k !}\left(\frac{z}{2}\right)^{2 k+v} ; \quad \Re(v)>-1, z \in \mathbb{C} .
$$

As for the historical background of related PDF and the associated cumulative distribution function (CDF) we consult the monographs [1,18]. In accordance with ([18] (Chapter 1, §5)) the PDF of $\xi \sim \chi_{n}^{\prime 2}(\lambda)$ was pioneered in 1928 by Fisher [19] by a limiting process, while the explicit derivation belongs to Tang [20] ten years later (we also draw the interested reader's attention to ([1] (Chapter 29, pp. 435 et seq.)). In 1949 Patnaik [21], then, among others, Pearson [22], Sankaran [23] and Temme [24] have been studied the $\chi_{n}^{\prime 2}(\lambda)$ distribution; Temme claimed that his formulae have certain computational advantages

$$
F_{n, \lambda}(x)=\left\{\begin{array}{lc}
1-\frac{1}{2}\left(\frac{x}{\lambda}\right)^{n / 4}\left[T_{n / 2-1}(\sqrt{\lambda x}, \omega)-\sqrt{\frac{\lambda}{x}} T_{n / 2}(\sqrt{\lambda x}, \omega)\right], & x>\lambda \\
\frac{1}{2}\left(\frac{x}{\lambda}\right)^{n / 4}\left[\sqrt{\frac{\lambda}{x}} T_{n / 2}(\sqrt{\lambda x}, \omega)-T_{n / 2-1}(\sqrt{\lambda x}, \omega)\right], & x<\lambda
\end{array},\right.
$$

where $\omega=\frac{1}{2}(\sqrt{x}-\sqrt{\lambda})^{2} / \sqrt{\lambda x}$ and

$$
T_{v}(\alpha, \omega)=\int_{\alpha}^{\infty} \mathrm{e}^{-(\omega+1) t} I_{v}(t) \mathrm{d} t
$$

Here we are interested in the CDF used in communication theory ([25] (p. 66, Equation (1.1)))

$$
F_{n, \lambda}(x)=1-Q_{n / 2}(\sqrt{\lambda}, \sqrt{x}), \quad x>0,
$$

where [26]

$$
Q_{v}(a, b)=\frac{1}{a^{v-1}} \int_{b}^{\infty} t^{v} \mathrm{e}^{-\left(t^{2}+a^{2}\right) / 2} I_{v-1}(a t) \mathrm{d} t, \quad a, v>0 ; b \geq 0,
$$

denotes the generalized Marcum $Q$-function of the order $v$.

Finally, it is worth mentioning that Brychkov recently published a closed expression for the generalized Marcum $Q$-function ([27] (p. 178, Equation (7))) in terms of the complementary error function $z \mapsto \operatorname{erfc}(z)$ ([28] (p. 160, Equation (7.2.2))), which immediately implies a new formula for $\mathrm{CDF}(3)$ in the case when $n \in \mathbb{N}$ is odd. In turn, in the case of an even number of the degrees of freedom, Jankov Maširević derived the following expression for the appropriate CDF for all $\lambda>0, x>0$ [25] 


$$
\begin{aligned}
F_{2 n, \lambda}(x)=1 & -\frac{\sqrt{\lambda x}}{2} I_{1}(\sqrt{\lambda x})\left[K_{0}(\sqrt{\lambda x})-K_{0}\left(\sqrt{\lambda x}, \ln \sqrt{\frac{x}{\lambda}}\right)\right] \\
& +\lambda I_{0}(\sqrt{\lambda x}) \frac{\partial}{\partial \lambda}\left[K_{0}(\sqrt{\lambda x})-K_{0}\left(\sqrt{\lambda x}, \ln \sqrt{\frac{x}{\lambda}}\right)\right] \\
& -\mathrm{e}^{-\frac{\lambda+x}{2}} \sqrt{\frac{\lambda}{x}} \sum_{m=1}^{n}\left(\sqrt{\frac{x}{\lambda}}\right)^{m} I_{m-1}(\sqrt{\lambda x})
\end{aligned}
$$

Here $K_{v}$ stands for the modified Bessel functions of the second kind and

$$
K_{v}(z, w)=\frac{\sqrt{\pi}}{\Gamma\left(v+\frac{1}{2}\right)}\left(\frac{z}{2}\right)^{v} \int_{0}^{w} \mathrm{e}^{-z \cosh t} \sinh ^{2 v} t \mathrm{~d} t, \quad \Re(v)>-1 / 2,
$$

is its incomplete variant ([29] (p. 26, Equation (1.30))), while

$$
\lim _{w \rightarrow \infty} K_{v}(z, w)=K_{v}(z), \quad \Re(z)>0,
$$

in the pointwise sense. Jankov Maširević established the computational efficiency of Expression (5) versus the formulae derived by Patnaik, and those by Temme for even $n \in \mathbb{N}$, concluding that her approach is more efficient, compare ([25] (Section 3)).

The main aim of this paper is to present new results for the CDF (3) concerning approximation formulae obtained by two variants of the second mean-value theorems for definite integrals. Throughout, the non-centrality parameter $\lambda>0$ and the variable $x>0$.

\section{Preliminaries and Auxiliary Results}

Combining the integral form of the Marcum $Q$-function (4) and the integral ([30] (p. 306, Equation (2.15.5.4)))

$$
\int_{0}^{\infty} t^{v+1} \mathrm{e}^{-p t^{2}} I_{v}(c t) \mathrm{d} t=\frac{c^{v} \mathrm{e}^{c^{2} /(4 p)}}{(2 p)^{v+1}}, \quad \Re(p)>0, \Re(v)>-1,|\arg (c)|<\pi,
$$

we express the CDF (3) for all $\lambda>0$ and $x>0$ as

$$
F_{n, \lambda}(x)=\frac{\mathrm{e}^{-\lambda / 2}}{\lambda^{n / 4-1 / 2}} \int_{0}^{\sqrt{x}} t^{n / 2} \mathrm{e}^{-t^{2} / 2} I_{n / 2-1}(\sqrt{\lambda} t) \mathrm{d} t
$$

This formula is the starting point for our main results, which concerns the approximate calculation of the involved integral using two different types of mean-value theorems.

Our next main tools are two mean-value theorems for integrals, of which the integrands contain products of two suitable functions $f, g$, say. Both theorems belong to the so-called second mean-value theorems for definite integrals. The ancestor results of the first version theorem belongs to Bonnet ([31] (p. 14)); however, for the second one we are referred to the memoir by du Bois-Reymond ([32] (p. 83)) or also to Hobson's article [31]. The case in which at least one of the input functions $f, g$ is a constant (first mean-value theorem) we skip in our present considerations. Now, recall the Bonnet variant of second mean-value theorem by Schwind-Ji-Koditschek.

Theorem 1. ([33] (p. 559, Theorem 2)). Suppose $f \in \mathrm{C}(a, b]$ and $g \geq 0$ is integrable on $(a, b)$. Let $x \in(a, b]$ be fixed. If both $\lim _{t \rightarrow a}(f(t)-K) /(t-a)^{r}$ and $\lim _{t \rightarrow a} g(t) /(t-a)^{s}$ exist and differ from zero for some constant $K$, a non-zero $r$ and some $s>-1$ with $r+s>-1$, then:

1. There exists $c_{x} \in(a, x]$ so, that

$$
\int_{a}^{x} f(t) g(t) \mathrm{d} t=f\left(c_{x}\right) \int_{a}^{x} g(t) \mathrm{d} t
$$


2. Moreover, for any such choice of $c_{x}$ there holds

$$
\lim _{x \rightarrow a} \frac{c_{x}-a}{x-a}=\left(\frac{s+1}{r+s+1}\right)^{\frac{1}{r}}
$$

Remark 1. We notice that often a good choice for $K$ in Theorem 1 is to take $K=\lim _{t \rightarrow a+} f(t)$ if the limit exists or $K=0$ otherwise, consult ([33] (p. 561)), also see [34-36] for ancestry of (7), which describes the asymptotic behavior of $c_{x}$.

Another approach in approximating the CDF of $\chi_{n}^{\prime 2}(\lambda)$ is based on the use of the Okamura's version of the du Bois-Reymond's second mean-value theorem for definite integrals $[37,38]$.

Theorem 2. ([39] (Equation (14))). Let $f:[a, b] \mapsto \mathbb{R}$ be monotone and $g:[a, b] \mapsto \mathbb{R}$ integrable. Then there exists a $c \in[a, b]$ such that

$$
\int_{a}^{b} f(t) g(t) \mathrm{d} t=f(a+) \int_{a}^{c} g(t) \mathrm{d} t+f(b-) \int_{c}^{b} g(t) \mathrm{d} t .
$$

We point out that both Theorems 1 and 2 hold for Riemann integrable input functions. However, stronger second mean-value theorem results for definite integrals for Lebesgue integrable functions have been presented by Wituła-Hetmaniok-Słota in ([40] (p. 1614, Theorem 3)).

\section{Approximating CDF of $\chi_{n}^{\prime 2}(\lambda)$ Distribution}

In this section we will state and prove our main results, derived from the formula (6) and the mean-value Theorems 1 and 2.

Theorem 3. Let $n \in \mathbb{N}, \lambda>0$ and $x>0$.

1. Then, there exists $c_{x} \in(0, \sqrt{x}]$ such that

$$
F_{n, \lambda}(x)=\left(\frac{x}{\lambda}\right)^{n / 4} \mathrm{e}^{-\frac{\lambda+c_{x}^{2}}{2}} I_{n / 2}(\sqrt{\lambda x})
$$

2. For $c_{x}$ there holds

$$
\lim _{x \rightarrow 0} \frac{c_{x}^{2}}{x}=\frac{n}{n+2}, \quad n \in \mathbb{N}
$$

while

$$
F_{n, \lambda}(x)=\frac{\mathrm{e}^{-\lambda / 2}}{\Gamma(n / 2+1)}\left(\frac{x}{2}\right)^{n / 2}(1+\mathscr{O}(x)), \quad x \rightarrow 0 .
$$

Proof. Consider the form of CDF given in (6). Making use of Theorem 1, with $f(t)=$ $\mathrm{e}^{-t^{2} / 2} \in \mathrm{C}\left(\mathbb{R}_{+}\right)$, which imply $K=\lim _{t \rightarrow 0} f(t)=1$ and by L'Hospital rule and $r=2$ follows

$$
\lim _{t \rightarrow 0} \frac{f(t)-K}{t^{r}}=\lim _{t \rightarrow 0} \frac{\mathrm{e}^{-t^{2} / 2}-1}{t^{2}}=-\frac{1}{2} \neq 0 ;
$$

then, choosing $g(t)=t^{n / 2} I_{n / 2-1}(t \sqrt{\lambda})$ and $s=n-1$ we have

$$
\lim _{t \rightarrow 0} \frac{g(t)}{t^{s}}=\lim _{t \rightarrow 0} \frac{I_{n / 2-1}(t \sqrt{\lambda})}{t^{n / 2-1}}=\frac{\lambda^{(n-2) / 4}}{2^{n / 2-1} \Gamma(n / 2)} \neq 0,
$$

bearing in mind the asymptotics of the modified Bessel $I_{v}$ for small $z \rightarrow 0$ which is the consequence of (2): 


$$
I_{v}(z)=\frac{1}{\Gamma(v+1)}\left(\frac{z}{2}\right)^{v}\left(1+\mathscr{O}\left(z^{2}\right)\right), \quad-v \notin \mathbb{N} .
$$

Hence, for $x>0$ fixed, according to part 1 of Theorem 1 , there exists a $c_{x} \in(0, \sqrt{x}]$ for which

$$
F_{n, \lambda}(x)=\frac{\mathrm{e}^{-\frac{\lambda+c_{x}^{2}}{2}}}{\lambda^{n / 4-1 / 2}} \int_{0}^{\sqrt{x}} t^{n / 2} I_{n / 2-1}(t \sqrt{\lambda}) \mathrm{d} t=\left(\frac{x}{\lambda}\right)^{n / 4} \mathrm{e}^{-\frac{\lambda+c_{x}^{2}}{2}} I_{n / 2}(\sqrt{\lambda x}),
$$

where in the last equality the formula ([41] (p. 676, Equation (6.561.7)))

$$
\int_{0}^{1} t^{v+1} I_{v}(a t) \mathrm{d} t=a^{-1} I_{v+1}(a), \quad \Re(v)>-1,
$$

was taken.

By the second part of Theorem 1 , bearing in mind that $c_{x} \in(0, \sqrt{x}]$ and setting $r=2, s=n-1, a=0$, we have

$$
\lim _{x \rightarrow 0} \frac{c_{x}^{2}}{(\sqrt{x})^{2}}=\lim _{x \rightarrow 0} \frac{c_{x}^{2}}{x}=\frac{n}{n+2}, \quad n \in \mathbb{N},
$$

that is (9). Now, the asymptotic behavior of the modified Bessel Function (11) approves the Relation (10).

Corollary 1. Let the situation be the same as in the preamble of Theorem 3. Then there exists $c=c_{x} \in(0,1]$ such that

$$
F_{n, \lambda}(x)=\left(\frac{x}{\lambda}\right)^{n / 4} \mathrm{e}^{-\frac{\lambda+x \cdot c^{2}}{2}} I_{n / 2}(\sqrt{\lambda x})
$$

Proof. Using the substitution $u=t / \sqrt{x}$, from (6) mutatis mutandis

$$
F_{n, \lambda}(x)=\sqrt{\lambda x} \mathrm{e}^{-\lambda / 2}\left(\frac{x}{\lambda}\right)^{n / 4} \int_{0}^{1} u^{n / 2} \mathrm{e}^{-x u^{2} / 2} I_{n / 2-1}(u \sqrt{\lambda x}) \mathrm{d} u,
$$

and then applying Theorem 1 repeating the above procedure for $f(u)=\mathrm{e}^{-x u^{2} / 2}, r=2$, $g(u)=u^{n / 2} I_{n / 2-1}(u \sqrt{\lambda x})$ and $s=n-1$ we readily conclude the Formula (12).

In what follows we propose some numerical approximations for the real number $c_{x}$ given in part 2 of Theorem 3 for small values of non-centrality parameter $\lambda>0$.

Corollary 2. Let $n \in \mathbb{N}$ and $x>0$. When $\lambda \rightarrow 0$, in (8) we have

$$
c_{x}^{2}=-2 \log \left[\left(\frac{2}{x}\right)^{n / 2} \gamma\left(\frac{n}{2}+1, \frac{x}{2}\right)+\mathrm{e}^{-x / 2}\right] .
$$

Proof. Combining the Formulae (3) and ([26] (p. 70))

$$
\lim _{a \rightarrow 0} Q_{v}(a, b)=\frac{1}{\Gamma(v)} \Gamma\left(v, b^{2} / 2\right)
$$

where $\Gamma(\cdot, \cdot)$ denotes the upper incomplete gamma function ([28] (p. 174, Equation (8.2.2)))

$$
\Gamma(a, z)=\int_{z}^{\infty} t^{a-1} \mathrm{e}^{-t} \mathrm{~d} t, \quad \Re(a)>0,
$$


we obtain

$$
\lim _{\lambda \rightarrow 0} F_{n, \lambda}(x)=1-\frac{\Gamma(n / 2, x / 2)}{\Gamma(n / 2)} .
$$

Now, from (11) we observe

$$
\lim _{\lambda \rightarrow 0} \frac{I_{n / 2}(\sqrt{\lambda x})}{\lambda^{n / 4}}=\frac{x^{n / 4}}{2^{n / 2} \Gamma(n / 2+1)}
$$

which, in conjunction with (8) implies that

$$
\lim _{\lambda \rightarrow 0} F_{n, \lambda}(x)=\left(\frac{x}{2}\right)^{n / 2} \frac{\mathrm{e}^{-c_{x}^{2} / 2}}{\Gamma(n / 2+1)}, \quad c_{x} \in(0, \sqrt{x}] .
$$

Equating the right-hand-side expressions in (15) and (16) we arrive at

$$
\Gamma(n / 2+1)-\frac{n}{2} \Gamma(n / 2, x / 2)=\left(\frac{x}{2}\right)^{n / 2} \mathrm{e}^{-c_{x}^{2} / 2} .
$$

The identities ([28] (p. 178, Equation (8.8.2-3)))

$$
\Gamma(a+1, z)=a \Gamma(a, z)+z^{a} \mathrm{e}^{-z} ; \quad \gamma(a, z)+\Gamma(a, z)=\Gamma(a),
$$

where $\gamma(\cdot, \cdot)$ is the lower incomplete gamma function, defined by ([28] (p. 174, Equation (8.2.1)))

$$
\gamma(a, z)=\int_{0}^{z} t^{a-1} \mathrm{e}^{-t} \mathrm{~d} t, \quad \Re(a)>0,
$$

one transforms (17) into

$$
\left(\frac{2}{x}\right)^{n / 2} \gamma(n / 2+1, x / 2)+\mathrm{e}^{-x / 2}=\mathrm{e}^{-c_{x}^{2} / 2} .
$$

Now, obvious steps lead to the final form of $c_{x}^{2}$.

Corollary 3. For the small enough values of the non-centrality parameter $\lambda$ and the argument $x$ the magnitude of approximation satisfies the relation

$$
\frac{c_{x}^{2}}{x}-\frac{n}{n+2}=-\frac{n x}{4(n+4)}+o(x), \quad x \rightarrow 0 .
$$

Proof. Recalling the asymptotic of the lower incomplete gamma function, which we deduce from the hypergeometric form expression ([17] (p. 262, Equation (6.5.12))), written in Landau's notation

$$
\gamma(\alpha, z)=\frac{z^{\alpha}}{\alpha}\left(1-\frac{\alpha z}{\alpha+1}+o(z)\right), \quad z \rightarrow 0,
$$

after asymptotic expansion of both expressions inside square brackets in (14), we get

$$
\begin{aligned}
\frac{c_{x}^{2}}{x} & =-\frac{2}{x} \log \left[\frac{x}{n+2}\left(1-\frac{(n+2) x}{2(n+4)}+o(x)\right)+1-\frac{x}{2}+\frac{x^{2}}{8}+o\left(x^{2}\right)\right] \\
& =-\frac{2}{x} \log \left[1-\frac{n x}{2(n+2)}+\frac{n x^{2}}{8(n+4)}+o\left(x^{2}\right)\right]
\end{aligned}
$$

For when $n$ is fixed and $x$ is small enough, it is legitimate to express the logarithm via its asymptotic expansion $\log (1+h)=h+o(h),|h|<1$, which approves (18). 
Remark 2. The associated limit result (18) enables the approximation

$$
F_{n, \lambda}(x) \simeq\left(\frac{x}{\lambda}\right)^{n / 4} \exp \left\{-\frac{\lambda}{2}-\frac{n x}{2(n+2)}+\frac{n x^{2}}{8(n+4)}\right\} I_{n / 2}(\sqrt{\lambda x}) .
$$

This estimate we can readily take into account in numerical calculation of CDF for the purpose of comparison with another representations like Patnaik's and Temme's, for instance.

Corollary 4. For all $\lambda>0, x>0$ we have

$$
F_{1, \lambda}(x)=\sqrt{\frac{2}{\pi \lambda}} \sinh (\sqrt{\lambda x}) \mathrm{e}^{-\frac{\lambda+c_{x}^{2}}{2}},
$$

where

$$
c_{x}^{2}=x+2 \log \left[1+\frac{1}{\lambda}-\sqrt{\frac{x}{\lambda}} \frac{\cosh (\sqrt{\lambda x})}{\sinh (\sqrt{\lambda x})}\right]
$$

Moreover,

$$
F_{2, \lambda}(x)=\sqrt{\frac{x}{\lambda}} \mathrm{e}^{-\frac{\lambda+c_{x}^{2}}{2}} I_{1}(\sqrt{\lambda x})
$$

where

$$
c_{x}^{2}=x+2 \log \left[1-\sqrt{\frac{x}{\lambda}} \frac{I_{2}(\sqrt{\lambda x})}{I_{1}(\sqrt{\lambda x})}\right] .
$$

Proof. Having in mind that for non-negative integer $m$ there holds ([27] (p. 178, Equation (7)))

$$
\begin{aligned}
Q_{m+1 / 2}(a, b)=\frac{1}{2} & {\left[\operatorname{erfc}\left(\frac{b-a}{\sqrt{2}}\right)+\operatorname{erfc}\left(\frac{b+a}{\sqrt{2}}\right)\right] } \\
& +\mathrm{e}^{-\left(a^{2}+b^{2}\right) / 2} \sum_{k=1}^{m}\left(\frac{b}{a}\right)^{k-1 / 2} I_{k-1 / 2}(a b),
\end{aligned}
$$

the Formula (3) for $n=1$ becomes

$$
F_{1, \lambda}(x)=\frac{1}{2}\left[\operatorname{erf}\left(\frac{\sqrt{x}-\sqrt{\lambda}}{\sqrt{2}}\right)+\operatorname{erf}\left(\frac{\sqrt{x}+\sqrt{\lambda}}{\sqrt{2}}\right)\right] .
$$

As $(\operatorname{erf}(z))^{\prime}=2 \mathrm{e}^{-z^{2}} / \sqrt{\pi}$, equating (21) and the Formula (8) and then deriving such equality with respect to $\lambda$ we get

$$
\mathrm{e}^{-x / 2}\left(\mathrm{e}^{-\sqrt{\lambda x}}-\mathrm{e}^{\sqrt{\lambda x}}\right)=\frac{2}{\lambda} \mathrm{e}^{-c_{x}^{2} / 2}(\sqrt{\lambda x} \cosh (\sqrt{\lambda x})-(1+\lambda) \sinh (\sqrt{\lambda x})) .
$$

Finally, the definition of hyperbolic sine implies (19).

The Formula (3) for $n=2$ becomes $F_{2, \lambda}(x)=1-Q(\sqrt{\lambda}, \sqrt{x})$ where $Q_{1}(a, b) \equiv Q(a, b)$ is the Marcum Q-function. Now, knowing that ([42] (p. 1221, Equation (5)))

$$
\frac{\partial Q(a, b)}{\partial a}=b I_{1}(a b) \mathrm{e}^{-\left(a^{2}+b^{2}\right) / 2}
$$

the first derivative of (8), with respect of $\lambda$ becomes

$$
-\frac{\sqrt{x}}{2 \sqrt{\lambda}} \mathrm{e}^{-(\lambda+x) / 2} I_{1}(\sqrt{\lambda x})=\sqrt{x} \mathrm{e}^{-\left(\lambda+c_{x}^{2}\right) / 2}\left[\frac{\sqrt{x}}{2 \lambda} I_{2}(\sqrt{\lambda x})-\frac{I_{1}(\sqrt{\lambda x})}{2 \sqrt{\lambda}}\right],
$$


that is

$$
\mathrm{e}^{-x / 2}=\mathrm{e}^{-c_{x}^{2} / 2}\left[1-\sqrt{\frac{x}{\lambda}} \frac{I_{2}(\sqrt{\lambda x})}{I_{1}(\sqrt{\lambda x})}\right],
$$

giving (20).

The second approach in approximating the CDF of $\chi_{n}^{\prime 2}(\lambda)$ is to apply Theorem 2.

Theorem 4. Let $\lambda>0, x>0$ and $R_{\rho}(n)=\left[(2 / n-1)_{+}, 2 / n+1\right)$, where $(a)_{+}=\max \{0, a\}$. Then for all $\rho \in R_{\rho}(1)=[1,3)$ there exists some $c \in[0,1]$ for which

$$
F_{1, \lambda}(x)=\frac{\mathrm{e}^{-\lambda / 2}}{\sqrt{\pi}}\left(\frac{x}{2}\right)^{(\rho-1) / 4} \cosh (\sqrt{\lambda x})\left[\gamma\left(\frac{3-\rho}{4}, \frac{x}{2}\right)-\gamma\left(\frac{3-\rho}{4}, \frac{x c^{2}}{2}\right)\right] .
$$

When $\rho \in R_{\rho}(2)=[0,2)$ there exists certain $c \in[0,1]$ that

$$
\begin{aligned}
F_{2, \lambda}(x)= & \mathrm{e}^{-\lambda / 2}\left(\frac{x}{2}\right)^{\frac{\rho}{2}}\left\{I_{0}(\sqrt{\lambda x}) \gamma(1-\rho / 2, x / 2)\right. \\
& \left.+\left[\delta_{\rho 0}-I_{0}(\sqrt{\lambda x})\right] \gamma\left(1-\rho / 2, x c^{2} / 2\right)\right\}
\end{aligned}
$$

where $\delta_{a b}$ stands for the Kronecker delta.

Moreover, for all $n \in \mathbb{N}_{3}=\{3,4, \ldots\}$ and $\rho \in R_{\rho}(n)$ there exists $c \in[0,1]$ such that

$$
\begin{aligned}
F_{n, \lambda}(x)= & \frac{\lambda^{(2-n) / 4}}{\sqrt{2}} \mathrm{e}^{-\lambda / 2}\left(\frac{x^{\rho}}{2^{\rho-1}}\right)^{n / 4} I_{n / 2-1}(\sqrt{\lambda x}) \\
& \times\left[\gamma\left(\frac{(1-\rho) n+2}{4}, \frac{x}{2}\right)-\gamma\left(\frac{(1-\rho) n+2}{4}, \frac{x c^{2}}{2}\right)\right] .
\end{aligned}
$$

We remark that the value of $c$ is not necessarily the same throughout.

Proof. Consider the CDF's integral representation (13) in which the integration domain is the unit interval $[0,1]$. Our intention is to specify the appropriate input functions $f, g$ in a simple way and by scaling only the exponent of the power term - the integrand contains a product of three functions- to prepare it for the use of Okamura's Theorem 2. Precisely, consider for some real $\rho$ (which range will be established later):

$$
f_{n, \rho}(t)=t^{\rho n / 2} I_{n / 2-1}(t \sqrt{\lambda x}) ; \quad g_{n, \rho}(t)=t^{(1-\rho) n / 2} \mathrm{e}^{-x t^{2} / 2} .
$$

From Formula (2) we can conclude that the function $I_{v}(x)$ increases monotonically for $v>0, x>0$. Therefore, $f_{n, \rho}(t)$, as a product of monotonically increasing functions, also monotonically increases. However, to establish the interconnection between the scaling parameter $\rho$ and the degrees of freedom $n$ we are forced to employ a more sophisticated approach. Namely, investigating the monotone behavior of $f_{n, \rho}(t), t \in(0,1]$ we start with

$$
f_{n, \rho}^{\prime}(t)=t^{\rho n / 2-1}\left\{[(\rho+1) n / 2-1] I_{n / 2-1}(t \sqrt{\lambda x})+t \sqrt{\lambda x} I_{n / 2}(t \sqrt{\lambda x})\right\} .
$$

The function $I_{v}$ is monotone decreasing with respect to the order, viz. ([43] (p. 220, Equation (2)))

$$
I_{\nu}(x)>I_{\mu}(x), \quad \mu>v \geq 0, x>0,
$$

also consult [44-46] regarding this question. So, evaluating (25) we get

$$
\begin{aligned}
f_{n, \rho}^{\prime}(t) & \geq t^{\rho n / 2-1}[(\rho+1) n / 2-1+t \sqrt{\lambda x}] I_{n / 2}(t \sqrt{\lambda x}) \\
& \geq t^{\rho n / 2-1}[(\rho+1) n / 2-1] I_{n / 2}(t \sqrt{\lambda x}),
\end{aligned}
$$


which is sufficient to see that $f_{n, \rho}^{\prime}(t)>0$ for $\rho>2 / n-1$ and also follows $f_{n, 2 / n-1}^{\prime}(t)>0$ directly from (25). On the other side we have

$$
\int_{0}^{1} g_{n, \rho}(t) \mathrm{d} t=\frac{1}{2}\left(\frac{x}{2}\right)^{[(\rho-1) n-2] / 4} \gamma([(1-\rho) n+2] / 4, x / 2) ;
$$

this expression makes sense for $\rho<1+2 / n$. Thus, having in mind the finiteness of $f_{n, \rho}(0+)$ and collecting all these constraints we infer that the range of the scaling parameter $\rho$ is the interval $R_{\rho}(n)=\left[(2 / n-1)_{+}, 2 / n+1\right)$.

Firstly, consider $\rho \in R_{\rho}(1)=[1,3)$ with the associated input functions

$$
\begin{aligned}
& f_{1, \rho}(t)=t^{\rho / 2} I_{-1 / 2}(t \sqrt{\lambda x})=\frac{\sqrt{2 / \pi}}{\sqrt[4]{\lambda x}} t^{(\rho-1) / 2} \cosh (t \sqrt{\lambda x}) \\
& g_{1, \rho}(t)=t^{(1-\rho) / 2} \mathrm{e}^{-x t^{2} / 2} .
\end{aligned}
$$

Being $\rho \geq 1$, the input limits are

$$
f_{1, \rho}(0+)=0 ; \quad f_{1, \rho}(1)=\frac{\sqrt{2 / \pi}}{\sqrt[4]{\lambda x}} \cosh (\sqrt{\lambda x}) .
$$

From (13) Okamura's Theorem 2 there follows (22).

The case $n=2, \rho \in R_{\rho}(2)=[0,2)$ works since $I_{0}(0)=1$. Ergo, we have two different solutions: when $\rho=0$ and, respectively, $\rho \in R_{\rho}(2) \backslash\{0\} \equiv(0,2)$. Indeed, since

$$
\begin{aligned}
f_{2,0}^{\prime}(t) & =\sqrt{\lambda x} I_{1}(t \sqrt{\lambda x})>0, \\
f_{2, \rho>0}^{\prime}(t) & =t^{\rho-1}\left[\rho I_{0}(t \sqrt{\lambda x})+t \sqrt{\lambda x} I_{1}(t \sqrt{\lambda x})\right]>0, \quad t \in(0,1],
\end{aligned}
$$

both $f_{2,0}(t)$ and $f_{2, p>0}(t)$ monotone increase for $t \in(0,1]$. The associated limits read

$$
f_{2, \rho}(0+)=\delta_{\rho 0}, \quad f_{2, \rho}(1)=I_{0}(\sqrt{\lambda x}) ; \quad \rho \in R_{\rho}(2),
$$

which leads to the master Formula (23) for the $\operatorname{CDF} F_{2, \lambda}(x)$.

It remains to see $n \in \mathbb{N}_{3}, \rho \in R_{\rho}(n)$. Knowing that $I_{v}(0)=0, \Re(v)>0$, we have vanishing $f_{n, \rho}(0+)=0$ for $\rho \geq 0$ and $f_{n, \rho}(1)=I_{n / 2-1}(\sqrt{\lambda x})$. By the monotonicity of $f_{n, \rho}(t)$ and the integration result (26) of $g_{n, \rho}(t)$ we get

$$
\begin{aligned}
F_{n, \lambda}(x)= & \sqrt{\frac{\lambda}{2 \mathrm{e}^{\lambda}}}\left(\frac{2}{\lambda}\right)^{n / 4}\left(\frac{x}{2}\right)^{\rho n / 4} I_{n / 2-1}(\sqrt{\lambda x}) \\
& \times\left[\gamma\left(\frac{(1-\rho) n+2}{4}, \frac{x}{2}\right)-\gamma\left(\frac{(1-\rho) n+2}{4}, \frac{x c^{2}}{2}\right)\right] .
\end{aligned}
$$

The rest is obvious. This completes the proof of the expression (24).

Remark 3. Let $\xi_{1}, \xi_{2}$ be independent random variables defined on a standard probability space $(\Omega, \mathscr{A}, \mathrm{P})$ having $\chi_{n_{1}}^{\prime 2}\left(\lambda_{1}\right), \chi_{n_{2}}^{\prime 2}\left(\lambda_{2}\right)$ distributions, respectively. Then the rv $\xi_{1}+\xi_{2} \sim \chi_{n}^{\prime 2}(\lambda)$, where $n=n_{1}+n_{2}$ and $\lambda=\lambda_{1}+\lambda_{2}$, see, e.g., ([18] ( $p$. 33, Teorema 27)). According to this relation we can consider $F_{2, \lambda}(x)$ as the $\mathrm{CDF}$ of the sum of two $\chi_{1}^{\prime 2}\left(\lambda_{j}\right), j=1,2$ distributed random variables where the linear combination $\lambda=\theta \lambda_{1}+(1-\theta) \lambda_{2}, \theta \in[0,1]$ occurs between their non-centrality parameters.

Moreover, the values $\theta=0,1$ correspond to the problem of obtaining the CDF using the property $\chi_{n}^{\prime 2}(\lambda)=\chi_{1}^{\prime 2}(\lambda)+\chi_{n-1}^{\prime 2}(0) \equiv \chi_{1}^{\prime 2}(\lambda)+\chi_{n-1}^{2}$, where the no-central and the central ros on the right are mutually independent, consult ([1] ( $p .436))$ and the related quotations therein. 
Corollary 5. Let $\lambda>0, x>0$. Then for all $n \in \mathbb{N}_{2}=\{2,3,4, \ldots\}$ there exists certain $c \in[0,1]$ such that

$$
F_{n, \lambda}(x)=\sqrt{\frac{\pi \lambda}{2}} \mathrm{e}^{-\lambda / 2}\left(\frac{x}{\lambda}\right)^{n / 4} I_{n / 2-1}(\sqrt{\lambda x})[\operatorname{erf}(\sqrt{x / 2})-\operatorname{erf}(c \sqrt{x / 2})] .
$$

Also, for all $n \in \mathbb{N}_{3}=\{3,4, \ldots\}$ there exists some $c \in[0,1]$ for which

$$
F_{n, \lambda}(x)=\mathrm{e}^{-\lambda / 2}\left(\frac{2}{\lambda}\right)^{(n-2) / 4} I_{n / 2-1}(\sqrt{\lambda x})\left[\gamma\left(\frac{n+2}{4}, \frac{x}{2}\right)-\gamma\left(\frac{n+2}{4}, \frac{x c^{2}}{2}\right)\right] .
$$

Proof. The first case occurs when $\rho=1$ in Theorem 4. From (27) we have

$$
f_{1,1}(t)=\frac{\sqrt{2 / \pi}}{\sqrt[4]{\lambda x}} \cosh (t \sqrt{\lambda x})
$$

which results in $f_{1,1}(0+)=\sqrt{2 / \pi} / \sqrt[4]{\lambda x}$. Hence, we consider $n \in \mathbb{N}_{2}$ in which case $f_{n, 1}(0+)=0$ and $f_{n, 1}(1)=I_{n / 2-1}(\sqrt{\lambda x})$. Additionally, from (25) it follows for all $n \in$ $\mathbb{N}_{2}, t \geq 0$ that

$$
f_{n, 1}^{\prime}(t)=t^{n / 2-1} I_{n / 2-1}(t \sqrt{\lambda x})(n-1)+\sqrt{\lambda x} t^{n / 2} I_{n / 2}(t \sqrt{\lambda x}) \geq 0 .
$$

So, $f_{n, 1}(t)$ monotone increases on $[0,1]$. Therefore

$$
\begin{aligned}
F_{n, \lambda}(x) & =\sqrt{\lambda x} \mathrm{e}^{-\lambda / 2}\left(\frac{x}{\lambda}\right)^{n / 4} I_{n / 2-1}(\sqrt{\lambda x}) \int_{c}^{1} \mathrm{e}^{-x t^{2} / 2} \mathrm{~d} t \\
& =\sqrt{\frac{\pi \lambda}{2}} \mathrm{e}^{-\lambda / 2}\left(\frac{x}{\lambda}\right)^{n / 4} I_{n / 2-1}(\sqrt{\lambda x})[\operatorname{erf}(\sqrt{x / 2})-\operatorname{erf}(c \sqrt{x / 2})] .
\end{aligned}
$$

Here, the notation of the error function (or probability integral)

$$
\operatorname{erf}(z)=\frac{2}{\sqrt{\pi}} \int_{0}^{z} \mathrm{e}^{-t^{2}} \mathrm{~d} t
$$

has been used.

Taking $\rho=0$ in Theorem 4, from (27) $f_{1,0}(t)=\frac{\sqrt{2 /(\pi t)}}{\sqrt[4]{\lambda x}} \cosh (t \sqrt{\lambda x})$, no right limit exists at zero, hence a fortiori $n>1$. Having in mind the observations stated in the proof of Theorem 4 for $n \in \mathbb{N}_{3}$ the Formula (29) follows immediately from (24), setting $\rho=0$.

Remark 4. Recalling the relation ([28] (p. 176, Equation (8.4.1)))

$$
\gamma(1 / 2, x)=\sqrt{\pi} \operatorname{erf}(\sqrt{x})
$$

the representation Formula (28) becomes

$$
F_{n, \lambda}(x)=\sqrt{\frac{\lambda}{2}} \mathrm{e}^{-\lambda / 2}\left(\frac{x}{\lambda}\right)^{n / 4} I_{n / 2-1}(\sqrt{\lambda x})\left[\gamma(1 / 2, x / 2)-\gamma\left(1 / 2, x c^{2} / 2\right)\right] .
$$

Author Contributions: The authors contributed equally to the manuscript and typed, read and approved the final version. All authors have read and agreed to the published version of the manuscript.

Funding: This research received no external funding.

Acknowledgments: The authors are grateful to the referees for careful reading of the first version of the manuscript and for helpful comments that finally encompass the article.

Conflicts of Interest: The authors declare no conflict of interest. 


\section{References}

1. Johnson, N.L.; Kotz, S.; Balakrishnan, N. Continuous Univariate Distributions; John Wiley \& Sons, Inc.: New York, NY, USA, 1995; Volume 2.

2. Robert, C. Modified Bessel functions and their applications in probability and statistics. Stat. Probab. Lett. 1990, 9, 155-161. [CrossRef]

3. Kamel, A.S.; Abdel-Samad, A.I. On the computation of non-central Chi-square distribution function. Commun. Stat. Simul. Comput. 1990, 19, 1279-1291. [CrossRef]

4. András, S.; Baricz, Á. Properties of the probability density function of the non-central chi-squared distribution. J. Math. Anal. Appl. 2008, 346, 395-402. [CrossRef]

5. Scharf, L.L. Statistical Signal Processing: Detection, Estimation, and Time Series Analysis; Addison-Wesley Publishing Co.: Boston, MA, USA, 1990.

6. Lancaster, H.O. Forerunners of the Pearson $\chi^{2}$. Aust. J. Stat. 1966, 8, 117-126. [CrossRef]

7. Bienaymé, I.J. Sur la probabilité des erreurs d'après la méthode des moindres carrés. Liouville's J. Math. Pures Appl. 1852, 17, 33-78.

8. Pearson, K. On a criterion that a given system of deviations from the probable in the case of a correlated system of variables is such that it can be reasonably supposed to have arisen from random sampling. Philos. Mag. 1900, 50, 157-175. [CrossRef]

9. Helmert, F.R. Über die Berechnung des wahrscheinlichen Fehlers aus einer endlichen Anzahl wehrer Beobachtungsfehler. Z. Math. Phys. 1875, 20, 300-303.

10. Helmert, F.R. Über die Wahrscheinlichkeit der Potenzsummen der Beobachtungsfehler und über einige damit im Zusammenhange stehende Fragen. Z. Math. Phys. 1876, 21, 192-218.

11. Kruskal, W.H. Helmert's distribution. Am. Math. Mon. 1946, 53, 435-438. [CrossRef]

12. Sheynin, O.B. Origin of the theory of errors. Nature 1966, 211, 1003-1004. [CrossRef]

13. Plackett, R.L. Karl Pearson and the Chi-Squared Test. Int. Stat. Rev. 1983, 51, 59-72. [CrossRef]

14. Kendall, M.G. Studies in the history of probability and statistics. XXVI. The work of Ernst Abbe. Biometrika 1971, 58, 369-373. [CrossRef]

15. Abbe, E. Über die Gesetzmässigkeit in der Vertheilung der Fehler bei Beobachtungsreihen; Dissertation zur Erlangung der Venia Docendi bei den Phyilosophischen Fakultät in Jena; Verlag Frommann: Jena, Germany, 1863.

16. Watson, G.N. A Treatise on the Theory of Bessel Functions; Cambridge University Press: London, UK, 1922.

17. Abramowitz, M.; Stegun, I.A. (Eds). Handbook of Mathematical Functions with Formulas, Graphs, and Mathematical Tables; Applied Mathematics Series 55; National Bureau of Standards: Washington, DC, USA, 1964; Reprinted by Dover Publications, New York, 1972.

18. Mihoc, G.; Craiu, V. Treatise on Mathematical Statistics, Sampling and Estimation; With an English Table of Contents; Editura Academiei Republicii Socialiste România: Bucureşti, Romania, 1976; Volume I. (In Romanian)

19. Fisher, R.A. The general sampling distribution of the multiple correlation coefficient. Proc. R. Soc. Lond. 1928, 121, 654-673.

20. Tang, P.C. The power function of the analysis of variance tests with tables and illustrations of their use. Stat. Res. Mem. Lond. 1938, 2, 126-149.

21. Patnaik, P.B. The non-central $\chi^{2}-$ and the F-distributions and their applications. Biometrika 1949, 36, 202-234. [CrossRef]

22. Pearson, E.S. Note on an approximation to the distribution of non-central $\chi^{2}$. Biometrika 1959, 46, 202-232. [CrossRef]

23. Sankaran, M. Approximations to the non-central chi-square distribution. Biometrika 1963, 50, 199-204. [CrossRef]

24. Temme, N.M. Asymptotic and numerical aspects of the non-central chi-square distribution. Comput. Math. Appl. 1993, 25, 55-63. [CrossRef]

25. Jankov Maširević, D. On new formulas for the cumulative distribution function of the non-central chi-square distribution. Mediterr. J. Math. 2017, 14, 66. [CrossRef]

26. András, S.; Baricz, Á.; Sun, Y. The generalized Marcum Q-function: An orthogonal polynomial approach. Acta Univ. Sapientiae Math. 2011, 3, 60-76.

27. Brychkov, Y.A. On some properties of the Marcum $Q$ function. Integral Transform. Spec. Funct. 2012, 23, 177-182. [CrossRef]

28. Olver, F.W.J.; Lozier, D.W.; Boisvert, R.F.; Clark, C.W. (Eds.) NIST Handbook of Mathematical Functions; NIST and Cambridge University Press: Cambridge, UK, 2010.

29. Agrest, M.M.; Maksimov, M.S. Theory of Incomplete Cylindrical Functions and Their Applications; Springer: New York, NY, USA, 1971.

30. Prudnikov, A.P.; Brychkov, Y.A.; Marichev, O.I. Special Functions; Integrals and Series; Gordon and Breach Science Publishers: New York, NY, USA, 1986; Volume 2.

31. Hobson, E.W. On the second mean-value theorem of the integral calculus. Trans. Am. Math. Soc. 1908, 2, 14-23. [CrossRef]

32. du, Bois-Reymond, P. Über die allgemeinen Eigenschaften der Klasse von Doppelintegralen, zu welcher das Fouriersche Doppelintegral gehört. J. Reine Angew. Math. 1868, 69, 65-108.

33. Schwind, W.J.; Ji, J.; Koditschek, D.E. A physically motivated further note on the mean-value theorem for integrals. Am. Math. Mon. 1999, 106, 559-564. [CrossRef]

34. Bao-Lin, Z. A note on the mean-value theorem for integrals. Am. Math. Mon. 1997, 104, 561-562. [CrossRef]

35. Jacobson, B. On the mean-value theorem for integrals. Am. Math. Mon. 1982, 89, 300-301. [CrossRef]

36. Polezzi, M. On the weighted mean value theorem for integrals. Internat. J. Math. Ed. Sci. Technol. 2006, 37, 868-870. [CrossRef]

37. Matsumoto, T. Hiroshi Okamura. Mem. Coll. Sci. Univ. Kyoto Ser. A Math. 1950, 26, 1-3. [CrossRef] 
38. Okamura, H. On the second mean-value theorem of integral. In Mathematics; Kyoto Mathematical Society: Kyoto, Japan, 1947; Volume 1. (In Japanese)

39. Baricz, Á.; Pogány, T.K. On a sum of modified Bessel functions. Mediterr. J. Math. 2014, 11, 349-360. [CrossRef]

40. Wituła, R.; Hetmaniok, E.; Słota, D. A stronger version of the second mean value theorem for integrals. Comput. Math. Appl. 2016, 64, 1612-1615. [CrossRef]

41. Gradshteyn, I.S.; Ryzhik, I.M. Table of Integrals, Series, and Products, 6th ed.; Jeffrey, A., Zwillinger, D., Eds.; Academic Press: New York, NY, USA, 2000.

42. Pratt, W.K. Partial differentials of Marcum's Q function. Proc. IEEE 1968, 56, 1220-1221. [CrossRef]

43. Jones, A.L. An extension of an inequality involving modified Bessel functions. J. Math. Phys. 1968, 47, 220-221. [CrossRef]

44. Cochran, J.A. The monotonicity of modified Bessel functions with respect to their order. J. Math. Phys. 1967, 46, 220-222. [CrossRef]

45. Nåsell, I. Inequalities for modified Bessel functions. Math. Comput. 1974, 28, 253-256. [CrossRef]

46. Soni, R.P. On an inequality for modified Bessel functions. J. Math. Phys. 1965, 44, 406-407. [CrossRef] 